\title{
Optimized Computerized Order Entry can Reduce Errors in Electronic Pre- scriptions and Associated Pharmacy Calls to Clarify (CTC)
}

Jaimin Patel ${ }^{1}$; Richard Ogletree ${ }^{2}$; Allison Sutterfield ${ }^{1}$; John C. Pace ${ }^{1}$; Laurene Lahr ${ }^{1}$

${ }^{1}$ University of Mississippi, Neonatology, Department of Information systems., Jackson, Mississippi, United States; 2University of Mississippi Medical Center, Pharmacy, Jackson, Mississippi, United States;

\section{Keywords}

Electronic prescribing, medication errors, CPOE, electronic health records, cost

\section{Summary}

Background: After implementation of a system-wide EMR within our university system, e-prescribing is now commonplace.

Objective: The authors conducted a study to assess whether optimization of computerized provider order entry (CPOE) can reduce errors in these electronically transmitted prescriptions and would require less frequent interventions from pharmacists, in particular the need for them to "call to clarify" (CTC) details of particular prescriptions. Secondary analysis based on cost assumptions was preformed to presume cost differences before and after optimization changes.

Materials and Methods: In order to generate complete, error-free prescriptions, optimization changes were implemented in the form of in line validation messages. These messages were generated if (1) an order did not specify a provider or pharmacy; (2) the DEA requirements were not met; (3) character limits were exceeded in patient sig or demographics or (4) administration instructions had breaks or had both discrete and free text elements. Retrospectively, prescriptions were randomly selected from a nine month period before and after implementing changes. These prescriptions were analyzed by a pharmacist and a nurse to identify types of errors that would require a CTC to a prescribing provider. Errors were compared statistically to determine effectiveness of changes pre and post optimization.

Results: A total of 602 prescriptions were analyzed; 301 before changes and 301 after changes. Of these prescriptions, $20.27 \%$ had errors before changes and $12.96 \%$ had errors after changes. The decline in the error rate was considered statistically significant for $p<0.05$. The cost savings were estimated at $\$ 76$ per 100 prescriptions for pharmacist and physician time-cost estimates combined. Conclusions: Implementing optimization changes to the CPOE resulted in a reduction in error rate requiring pharmacist CTC. This study identifies effective optimization changes for electronic prescribing that can reduce prescribing errors and may result in cost saving.

\section{Correspondence to:}

Jaimin Patel

Assistant Prof. Pediatric, Assistant CMIO

University of Mississippi

Neonatology Division

W112, 2500 N. State St

Jckson, MS 39110.

Email: jpatel@umc.edu

\author{
Appl Clin Inform 2016; 7: 587-595 \\ http://dx.doi.org/10.4338/ACl-2015-10-RA-0140 \\ received: October 16, 2015 \\ accepted: April 18, 2016 \\ published: June 29, 2016 \\ Citation: Patel J, Ogletree R, Sutterfield A, Pace JC, Lahr \\ L. Optimized computerized order entry can reduce errors \\ in electronic prescriptions and associated pharmacy \\ calls to clarify (CTC). Appl Clin Inform 2016; 7: 587-595 \\ http://dx.doi.org/10.4338/ACl-2015-10-RA-0140
}




\section{Background}

Medication errors are reported to be responsible for more than 7000 deaths annually [1]. The adoption of computerized provider order entry (CPOE) systems can reduce medication errors [2]. E-prescribing has been widely adopted and has been shown to reduce the need for manual prescription entry. However, pharmacy staff still frequently must complete or edit certain fields, particularly drug name and patient instructions [3].

E-prescribing reduces manual prescription entry by the healthcare provider, but it can still result in patient safety concerns. These safety concerns include validity and completeness of the information regarding medications, accidentally prescribing duplicate medications, and selecting the wrong drug or dose due to drop-down menus and small screen size [4]. On average, pharmacist may have to intervene $3.8 \%$ of the time for e-prescription. This percentage increases with new prescriptions (4.1\%) due to errors of omission, dosing etc [5]. The most common intervention by pharmacists is contacting the prescriber (64.1\%) for information verification. Such action requires an average of 6.07 minutes to conduct their intervention and an incremental dispensing cost of \$4.74 [5].

Implementing CPOE can reduce medication errors, but optimizing CPOE systems may also significantly improve performance. In this study, we aim to analyze how optimization of CPOE can further reduce the error rate. The guidelines published by SureScripts [6] may result in improved error rates by transmitting more complete prescriptions.

\section{Objectives}

The objective of this study is to identify the most common types of errors seen by pharmacists in electronically transmitted prescriptions as well as to investigate the effects of implementation of optimization changes in $\mathrm{CPOE}$ on reduction of such errors.

\section{Material and Methods}

\subsection{Inclusion criteria}

Randomly selected e-prescriptions were extracted from Epic EMR nine months prior to implementing changes (Jan 2013-Sep 2013) and nine months after implementing changes (Oct 2013 to June 2014). This random sample was drawn from the inpatient and outpatient departments of all potentially affected clinics and hospitals at the University of Mississippi Medical Center.

\subsection{Exclusion criteria}

Prescriptions were excluded if they were written manually, or if the information could not be extracted. E-prescriptions were excluded if they were not transmitted due to technical reasons. Also, written and held prescriptions which were not actually transmitted were also excluded from the study. In order to fulfill criteria for exclusion, selected prescription were selected from EMR prescriptions which had completed the electronic transmission process.

\subsection{Study design}

This study was done retrospectively, to determine effectiveness of the optimization changes that were implemented in Epic-EMR CPOE in September 2013.

The changes were determined appropriate based upon the guidelines published by SureScripts [6].

In-Line Validation Messages were generated if the e-prescription order:

1. Did not have e-prescribing provider

2. Did not have pharmacy specified

3. Did not meet DEA requirements 
4. Contained line breaks in comments for patient sig (administration instruction)

5. Exceeded the character limit for patient sig (administration instruction)

6. Contained both discrete and free text elements in patient sig. (administration instruction)

7. Exceeded the character limit within patient demographics

Optimization changes were implemented in the EMR across all specialties at University of Mississippi Medical Center.

The project team consisted of one physician, one pharmacist, one nurse and two electronic medical record analysts. Data was retrospectively collected by querying Epic EMR for a random selection of prescriptions for nine month period pre and post implementation of changes. A total of 602 prescriptions; 301 prescriptions prior to changes and 301 after implementing changes were extracted. All prescriptions were analyzed by a pharmacist (PharmD) and a nurse to identify the common types of errors that may require CTC with the prescribing provider. The pharmacist and nurse were not blinded to the status of the prescription in the study. Subsequently, prescriptions were divided into pre- and post- optimization groups. errors were classified and compared between the two groups.

\subsection{Statistical Methodology}

Analysis by a statistician revealed that using two tailed model, 270 prescriptions need to be included in each group to detect a decrease in CTC from $25 \%$ to $15 \%$ for $99 \%$ power and a P-value of 0.05 .

Extracted data about the prescriptions before and after optimization was analyzed by the pharmacist for error rate which was defined as a need to call the provider for clarification. By forming a contingency table $(2 \times 2)$, the error rate was compared using risk ratios, odds ratio and chi square test. Statistical calculations were completed using the website http://vassarstats.net/odds2x2.html.

\subsection{Cost calculations}

Cost calculations were performed based upon the following assumptions: 5 minute of call duration and salaries $\$ 200,000$ and $\$ 100,000$ for the physician and pharmacist respectively. The cost of the pharmacist's time was estimated at about $\$ 3.47$ per call $\{5$ min spent $\} \times\{(100000$ estimated salary)/(60 min X 8 hrs X 25 days X 12 months $)\}$ and the cost of the physician's time was estimated at about $\$ 6.94$ per call. According to one published study, contacting the prescriber by pharmacists for information verification requires an average of 6.07 minutes and has an incremental dispensing cost of $\$ 4.74$ [5]. We wanted to do a conservative cost analysis hence the use of conservative numbers in this study. Also there are potential additional costs related to pharmacy techs, physician assistants and nurses who may have been involved in the process. However, in this conservative analysis, those variables were not taken into account.

\section{Results}

University of Mississippi Medical Center is the only academic medical center in Mississippi. Epic EMR was implemented at University of Mississippi in June 2012. A total of 205,446 prescriptions were e-prescribed during pre-implementation period of Jan 2013 to Sep 2013, compared to 334,118 total e-prescriptions from Oct 2013 to June 2014. These numbers included prescriptions written for adults and children in both outpatient and inpatient settings. The median age of the patient population was 50 years, and the average age was 45.01 and 44.63 years respectively in each group prepost- implementation.

In this study, 301 randomly selected prescriptions in each group, pre- and post-implementation, were extracted from the EMR. Medication errors were identified at the discretion of the pharmacist and the nurse where a CTC was indicated based upon the information transmitted in the e-prescription. Afterwards, they were classified as pre and post optimization prescriptions.

The pharmacist and nurse determined there were errors in 61/301 (20.27\%) and 39/301 (12.96\%) of prescriptions pre- and post- optimization. See $>$ Figure 1 . This figure shows the total number of 
e-prescriptions in each period, the number of randomly selected prescriptions in each group and the number of prescriptions with errors in each group. This change in the number of errors was statistically significant with Pearson chi-square of $5.8(\mathrm{p}=0.016, \mathrm{p}<0.05)$. These errors were classified in 10 different categories and the data presented in $>$ Figure 2.

Based upon cost estimate of $\$ 3.47$ and $\$ 6.94$ per call for the pharmacist and physician respectively; there was an estimated $\$ 76$ cost savings per 100 prescriptions. $\{20.27-12.96=7.31$ (no of reduced calls per 100 prescriptions) x 10.41 (total of $3.47+6.94)=76\}$.

Two way sensitivity analysis was performed keeping actual cost variations in mind. See $>$ Table 1 for total cost estimates based upon varying number of reduced errors per 100 prescriptions along with variations in cost estimates of all involved healthcare providers' time per prescription error.

\section{Discussion}

In this study we identify optimization changes for electronic prescription system that could reduce errors by $7.31 \%(\mathrm{p}<0.05)$ that require a CTC. We also propose that this may ultimately result in cost savings.

\subsection{CPOE and varying error rate reduction may be due to varying de- sign of CPOE systems. Are these optimization changes at the root of varying error rates?}

Previous studies have reported variable results on error reduction after CPOE implementation. While most studies report a reduction of errors after CPOE implementation [7-15]; some authors have reported that CPOE may increase the errors [16]. Studies showing reductions in medication errors have reported variable results. [2]. Since there is wide variation between different CPOE's, there is a need to identify features/optimization changes that affect the error rate within different CPOE systems and to adopt those features as basic standards for future development and implementation. In this study we propose optimization changes that may reduce e-prescribing errors.

\subsection{Why total number of e-prescriptions increased between pre and post optimization groups?}

The significant increase in number of e-prescriptions $(205,446$ vs 334,118$)$ between the two study periods may have resulted from changes that were implemented. Particularly, creating in line messaging if provider or pharmacy information was missing, if the medication did not meet DEA requirements and if patient demographic exceeded e-prescribing character limit, would result in a provider being able to successfully e-prescribe and more likely to e-prescribe in future. Also, since EMR was recently implemented, it is possible that providers are becoming more comfortable with e-prescribing leading to an increased number of e-prescriptions. This study is limited by the fact that we did not analyze the number of failed e-prescription attempts.

\subsection{Why error rate differs between previously reported and current study?}

Different definitions of medication errors can be found in the literature. In this study we defined medication errors by whether or not the pharmacist felt a need for a CTC. Some studies define medication errors as events with potential to cause patient harm, while others count errors corrected by the hospital's internal redundant system auditing before reaching the patient. In this study we adopted the latter, since the aim of the study was to reduce the redundancy of the system and minimize the corrections needed before e-prescriptions were transmitted to the pharmacy. Our data estimates the error rate to be $20.27 \%$ and $12.96 \%$ pre and post optimization which is much higher than 
error rate reported in other studies. Error rates of $2.32-3.91 \%$ on handwritten and $0.82-0.69 \%$ on computer assisted prescriptions have been reported in an emergency department [9]. Another outpatient study found the error rate to be $7.6 \%$ for prescribing errors. Out of these prescribing errors $4.3 \%$ errors were at basic computerized sites vs $11 \%$ errors at handwritten prescription sites.[17]. This error rate discrepancy between our study and other studies may be due to the stricter definition of error used in our study. For example, if a dermatological cream was prescribed, but the affected area to be applied was not clarified in e-prescription, we counted it as an error. Also, we included multiple sites in a large academic center including inpatient, outpatient, and emergency room rather than studying a single department or setting.

\subsection{Cost saving estimates- Are they underestimated or overestimated?}

Considering the time spent by the pharmacist and physician for such phone calls, estimating at least 5 minutes spent by each, we estimated a cost saving of $\$ 76$ per 100 prescriptions. Additional costs may be incurred from other individuals who might be involved in the medication prescription process such as nurses, physician assistants and pharmacy technicians. Thus it is likely that we have underestimated actual costs. Also, we assumed the pharmacist would call a physician for each error detected. In actual practice, the pharmacist may correct some errors without calling the provider. In this case we may have overestimated costs. The cost calculations in this study were done to illustrate potential cost savings. Further studies are needed to determine if these cost savings are accurate.

The effects of e-prescribing on physician work load was not studied. Electronic prescriptions may have increased work load since even simple prescriptions require multiple field inputs. In that case we may have overestimated cost savings. However, the opposite may be true. Physician work load may be decreased if the pharmacist is not required to call multiple times per day to clarify prescription errors. The time lost due to interruptions in work flow in addition to spending time on phone was not taken into account; thus, cost savings may have been underestimated. Such variables were not taken into account.

\subsection{Explaining changes in the errors between two groups}

The most significant effects in error reduction or elimination were noted in the area of patient instruction, elimination of contrasting sig and dose clarifications. Since the provider was presented with in-line messages if the prescription sig contained line breaks, if it exceeded the character limit or if it contained both discrete and free text elements; these changes may have forced providers to clarify instructions, reducing the above mentioned errors.

Since there were no changes directly affecting the route of medication (for example in one instance IV formulation was prescribed PO and needed clarification) and forcing providers to clarify time of the day when medication should be taken (for example, sedatives are best taken in the evening, while the provider prescribed it "once"), those errors were not affected by optimization changes.

Mainly when dermatological creams were prescribed, the location were cream should be applied was not clarified (although that should be self-understood by patients) and since no changes were implemented to make location a required field it did not eliminate these types of errors. The reported increase in errors may have been due to random selection of these type of dermatological prescriptions. (i.e. random error). Also, there was no discrete field to state whether the patient is instructed to continue medications after using the prescribed quantity. The pharmacist would need to clarify the duration of treatment in this case. These type of errors were not affected by any of the changes implemented.

Only in the post optimization group is there an error requiring drug/formulation clarification. In this error, the provider prescribed dextromethorphan instead of a typical over the counter combination cold remedy requiring additional clarification. Random selection bias is suspected in uncovering this error. 


\subsection{Limitations of study}

This study is limited in several aspects. It is a retrospective study, and the team was not blinded. Since this was newly implemented e-prescription system, background error rate was not available prior to the study. Also, costs associated with phone calls including durations, were estimated and additional personnel such as nurses, pharmacy technicians, physician assistant who may have been involved in the process were not accounted for.

This study does not address the effect of a newly implemented EMR on the work load of busy providers and how this may have affected error rates.

With these limitations, this study was able to show potential improvements in errors by optimizing e-prescription CPOE. Further prospective randomized double blinded studies are needed to prove our hypothesis.

\section{Conclusions}

Optimizing CPOE by implementing described changes may result in a significant reduction in error rate requiring pharmacist CTC. This study also identifies other opportunities to optimize the process of electronic prescribing by adding "location" as required field in dermatological prescriptions and restricting appropriate route of medications thus preventing accidental prescription with wrong route of administration for medication.

\section{Clinical Relevance Statement}

E-prescription optimization changes in the CPOE may result in significant improvement in quality of prescriptions electronically transmitted to pharmacies and thus ultimately result in reduced time for calls to clarify prescriptions required by the pharmacists.

\section{Conflict of Interest}

The authors declare that they have no conflicts of interest in the research.

\section{Human Subjects Protection}

This study was performed in compliance with the World Medical Association Declaration of Helsinki on Ethical Principles for Medical Research Involving Human Subjects, and was reviewed by the University of Mississippi Institutional Review Board.

\section{Acknowledgements}

Authors thank the developers and pharmacists who worked on the project and helped to extract data. 


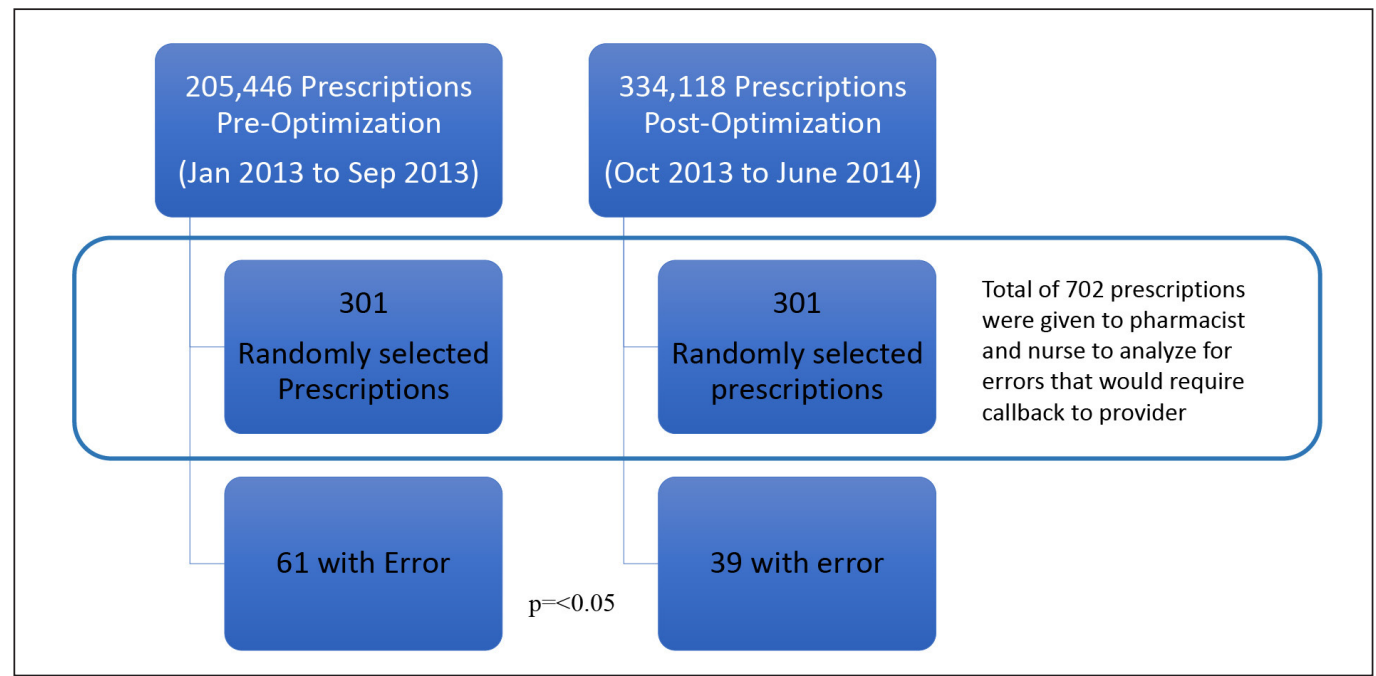

Fig. 1 Total number of prescriptions, number of randomly selected prescriptions and number of prescriptions with errors in each group.

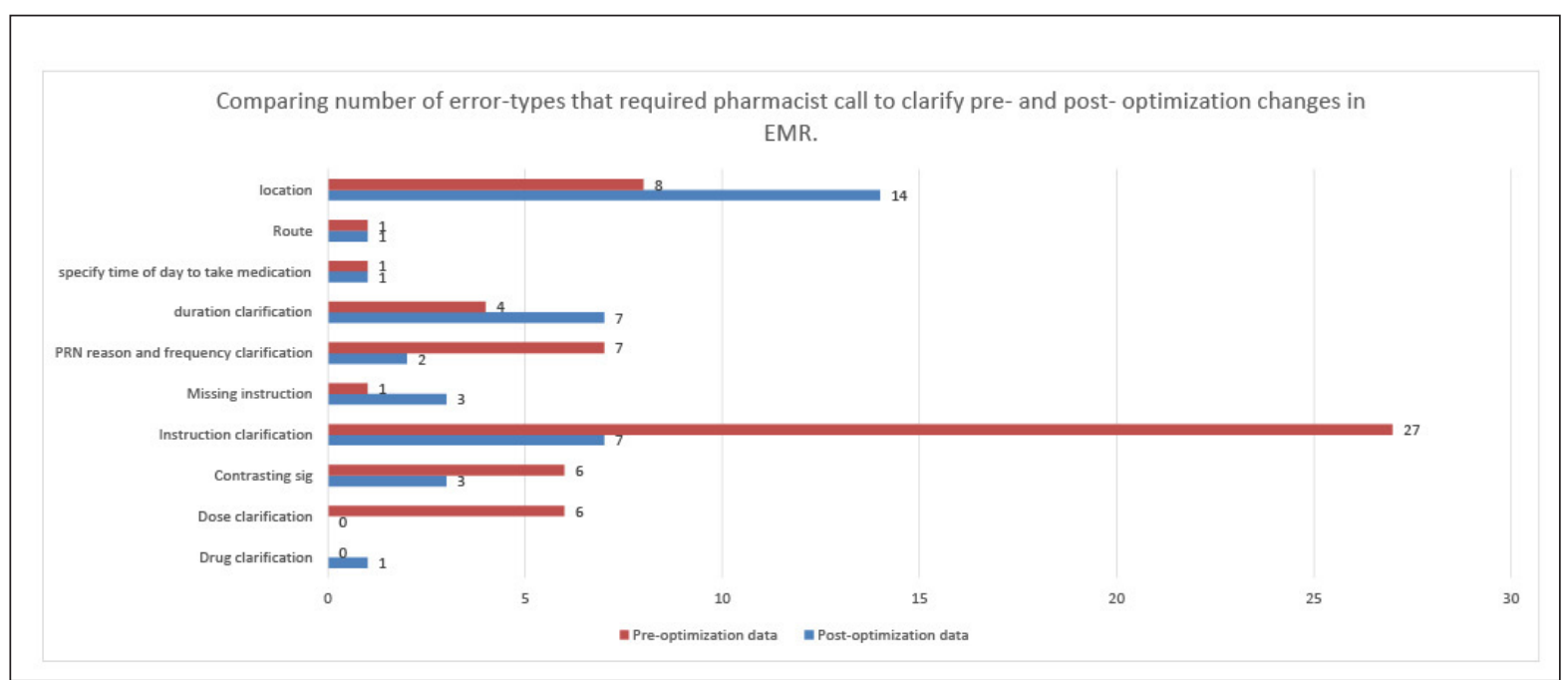

Fig. 2 Comparing number of error-types that required pharmacist call to clarify pre- and post- optimization changes in EMR. 
Table 1 Two way sensitivity analysis based on variable cost estimates per prescriptions and variable percent error reduction.

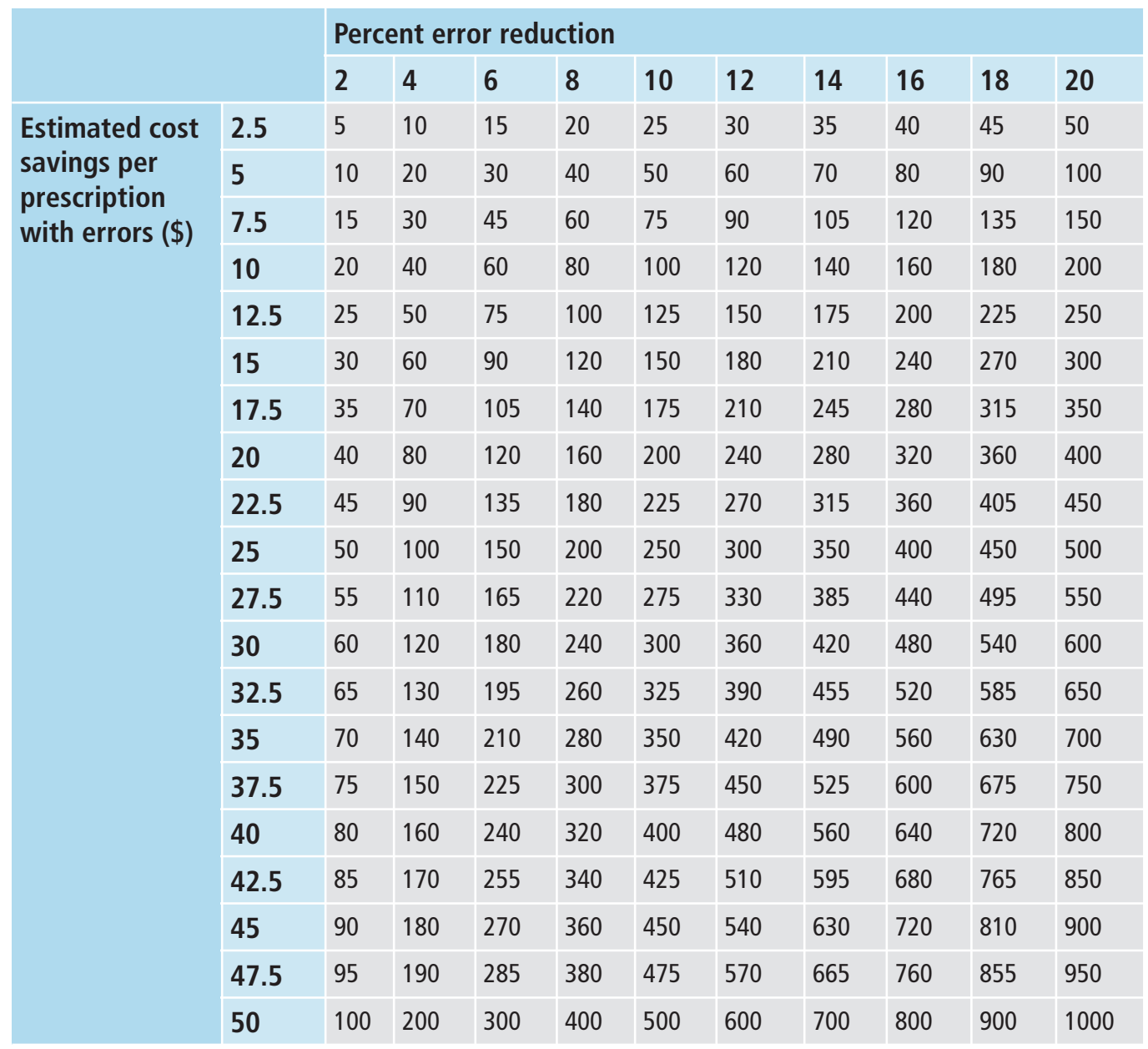




\section{References}

1. Manasse HR. Increase in US medicatlon-error deaths (Letter). Lancet 1998; 351(9116): 1655.

2. Radley DC, Wasserman MR, Olsho LE, Shoemaker SJ, Spranca MD, Bradshaw B. Reduction in medication errors in hospitals due to adoption of computerized provider order entry systems. J Am Med Inform Assoc 2013; 20(3): 470-476.

3. Grossman JM, Cross DA, Boukus ER, Cohen GR. Transmitting and processing electronic prescriptions: experiences of physician practices and pharmacies. Journal of the American Medical Informatics Association 2012; 19(3): 353-359.

4. Lapane KL, Waring ME, Dubé C, Schneider KL. E-Prescribing and Patient Safety: Results From a Mixed Method Study. Am J Pharm Benefits 2011; 3(2): e24-e34.

5. Warholak TL, Rupp MT. Analysis of community chain pharmacists' interventions on electronic prescriptions. J Am Pharm Assoc (2003) 2009; 49(1): 59-64. Available from: http://japha.org/article.aspx?doi=10.1331/JAPhA.2009.08013

6. Whittemore K. Guidelines for Creating High-Quality Electronic Prescriptions In The Ambulatory Healthcare Setting. http://surescripts.com. 2010. p. 1-18. Available from: http://surescripts.com/docs/defaultsource/Resources/quality_e-prescription_guidelines_surescripts.pdf

7. Bates DW, Teich JM, Lee J, Seger D, Kuperman GJ, Ma'Luf N, Boyle D, Leape L. The Impact of Computerized Physician Order Entry on Medication Error Prevention. J Am Med Informatics Assoc 1999; 6(4): 313-321.

8. Taylor J a, Loan L a, Kamara J, Blackburn S, Whitney D. Medication administration variances before and after implementation of computerized physician order entry in a neonatal intensive care unit. Pediatrics 2008; 121(1): 123-128.

9. Bizovi KE, Beckley BE, McDade MC, Adams AL, Lowe R a., Zechnich AD, Hedges JR. The Effect of Computer-assisted Prescription Writing on Emergency Department Prescription Errors. Acad Emerg Med 2002; 9(11): 1168-1175.

10. Cordero L, Kuehn L, Kumar RR, Mekhjian HS. Impact of computerized physician order entry on clinical practice in a newborn intensive care unit. J Perinatol 2004; 24(2): 88-93.

11. Evans RS, Pestotnik SL, Classen DC, Clemmer TP, Weaver LK, Orme JF, Lloyd JF, Burke JP. A computerassisted management program for antibiotics and other antiinfective agents. N Engl J Med 1998; 338(4): $232-238$.

12. Igboechi CA, Ng CL, Yang CS, Buckner AN. Impact of computerized prescriber order entry on medication errors at an acute tertiary care hospital. Hosp Pharm Thomas Land 2003; 38(3): 227-231.

13. Kim GR, Chen AR, Arceci RJ, Mitchell SH, Kokoszka KM, Daniel D, Lehmann CU. Error reduction in pediatric chemotherapy: computerized order entry and failure modes and effects analysis. Arch Pediatr Adolesc Med 2006; 160(5): 495-498.

14. Mahoney CD, Berard-Collins CM, Coleman R, Amaral JF, Cotter CM. Effects of an integrated clinical information system on medication safety in a multi-hospital setting. Am J Health Syst Pharm 2007; 64(18): 1969-1977.

15. Walsh KE, Landrigan CP, Adams WG, Vinci RJ, Chessare JB, Cooper MR, Hebert PM, Schainker EG, McLaughlin TJ, Bauchner H. Effect of computer order entry on prevention of serious medication errors in hospitalized children. Pediatrics 2008; 121(3): e421-e427.

16. Bradley VM, Steltenkamp CL, Hite KB. Evaluation of reported medication errors before and after implementation of computerized practitioner order entry. J Healthc Inf Manag 2006; 20(4): 46-53.

17. Gandhi TK, Weingart SN, Seger AC, Borus J, Burdick E, Poon EG, Leape LL, Bates DW. Outpatient prescribing errors and the impact of computerized prescribing. J Gen Intern Med 2005; 20(9): 837-841. 